\section{Description of an orthostatic form of myoclonus in 15 patients}

Myoclonus can be classified according to the physical activity with which myoclonic jerks are associated. Glass and colleagues have now proposed the new category of 'orthostatic' myoclonus (in which myoclonic bursts are associated with the action of standing upright), as distinct from 'action' or 'postural' myoclonus. In a recent paper, they report a retrospective review of the records of all 15 patients who were diagnosed with orthostatic myoclonus at the Mayo Clinic, Rochester, MN, during the period 2002-2006.

Of the 15 patients, 7 had a degenerative disorder of the CNS, 2 had a systemic illness, and the remaining 6 had no identifiable CNS disease causing myoclonus. Thirteen of the cases had initially been identified after patients presented with inadequately explained, slowly progressive gait dysfunction. 'Leg-jerking' was noted by the patient or examining physician in 13 cases. The myoclonus had distinct physiological characteristics in all 15 patients. No myoclonic bursts were observed on surface electromyography in any of the patients while they were at rest; sustained limb posture produced action myoclonus in some patients while seated. In all patients, however, myoclonic bursts became apparent, or increased in frequency, in multiple leg muscles immediately after standing. The myoclonus was easily distinguished from orthostatic tremor by the presence of nonrhythmic bursts and the absence of high-frequency discharges.

The authors conclude that orthostatic myoclonus is a distinct clinical entity, and might be a manifestation of a number of different CNS diseases. The underlying physiology of orthostatic myoclonus contributing to gait disturbance in the elderly remains unknown.

Original article Glass GA et al. (2007) Orthostatic myoclonus: a contributor to gait decline in selected elderly. Neurology 68: 1826-1830

\section{Occipital nerve stimulation improves medically refractory chronic cluster headache}

Cluster headache is a type of primary headache defined by bouts of very severe pain that occur several times daily. The chronic form of the condition can be medically intractable, forcing treating physicians to resort to invasive or neurally destructive surgical interventions that are associated with substantial morbidity. Peripheral stimulation of the occipital nerve has been successfully used in open-label trials to manage other forms of medically refractory headache.

Burns et al. investigated the use of bilateral occipital nerve stimulator implantation in a longterm, open-label study of eight patients with medically intractable chronic cluster headache (median age at surgery 46 years; median headache duration 6 years). Following implantation, six of the eight patients reported a meaningful improvement in the frequency or severity of their attacks after a median follow-up of 20 months (range 8-27 months) and stated that they would recommend the treatment to other similarly affected patients. The median level of improvement reported by these six patients was $60 \%$; two reported an improvement of $90 \%$ or more, but none became pain free. Complications associated with the occipital nerve stimulator included battery failure (five incidents), electrode failure or migration (four incidents), muscle recruitment, neck spasms, and electric shock sensations. Headache attacks returned when technical problems halted the stimulation.

The authors concluded that occipital nerve stimulation is a safe and effective treatment for cluster headache, but further studies, with the addition of a placebo control, will be necessary to establish the optimum stimulation amplitude, frequency, and pulse width for maximum stimulation efficacy.

Original article Burns B et al. (2007) Treatment of medically intractable cluster headache by occipital nerve stimulation: long-term follow-up of eight patients. Lancet 369: 1099-1106

\section{Microglial activation is a marker of presymptomatic HD}

Researchers have demonstrated that there is early and widespread activation of microglia in Huntington's disease (HD) presymptomatic gene carriers (PGCs), and have shown a strong relationship between this process and striatal neuronal dysfunction. They have also established the utility of the PET tracer ${ }^{11} \mathrm{C}-(\mathrm{R})-\mathrm{PK} 11195$ (PK) as a marker of subclinical disease progression in HD. PK binds selectively to the peripheral benzodiazepine binding sites expressed by 